\title{
Design of $\mathrm{Bi}-2223 / \mathrm{Ag}$ coil based on genetic algorithm and finite element method
}

\section{$\operatorname{AUTHOR}(\mathrm{S})$ :}

Higashikawa, K; Nakamura, T; Hoshino, T; Muta, I

\section{CITATION:}

Higashikawa, K ... [et al]. Design of Bi-2223/Ag coil based on genetic algo rithm and finite element method. IEEE TRANSACTIONS ON APPLIED SUPERCONDUCTIVITY 2005, 15(2): 1895-1898

\section{ISSUE DATE:}

2005-06

URL:

http://hdl.handle.net/2433/39960

\section{RIGHT:}

(c)2005 IEEE. Personal use of this material is permitted. However, permission to reprint/republish this material for advertising or promotional purposes or for creating new collective works for resale or redistribution to servers or lists, or to reuse any copyrighted component of this work in other works must be obtained from the IEEE. 


\title{
Design of Bi-2223/Ag Coil Based on Genetic Algorithm and Finite Element Method
}

\author{
Kohei Higashikawa, Taketsune Nakamura, Tsutomu Hoshino, and Itsuya Muta
}

\begin{abstract}
We designed Bi-2223/Ag coils based on genetic algorithm and finite element method. The winding geometry as well as the operating current of the coil was optimized for the minimization of its loss, on condition that its operating temperature, stored energy and total length of the conductor were constant. The loss was calculated with the use of the analytical expressions that could quantitatively describe the current transport characteristics in high $T_{\mathrm{c}}$ superconductor including their magnetic anisotropy and temperature dependence. Some optimized results were shown and discussed in this paper.
\end{abstract}

Index Terms-Finite element method, genetic algorithm, HTS coil, magnetic anisotropy, optimal design.

\section{INTRODUCTION}

$\mathbf{S}$ UPERCONDUCTING magnetic energy storage (SMES) is one of the strongest candidates for power quality control. Especially, high $T_{\mathrm{c}}$ superconducting (HTS) SMES is expected to lead to miniaturization as well as cost reduction compared to low $T_{\mathrm{c}}$ superconducting (LTS) counterpart. In such application, it is essential to optimize the winding geometry of the HTS coil because the performance of the coil is heavily dependent on its configuration due to the magnetic anisotropy of the current transport characteristics in the HTS conductor [1], [2].

There have been some reports on the design method of the HTS coil [3]-[8]. That is, the minimization of the volume or the amount of the conductor in the coil have been performed through the evaluation of the current transport performance of the coil. In these reports, however, the current transport characteristics in the conductor are numerically approximated with the use of experimental results.

On the other hand, we have already derived the analytical expressions of current density, $J$, versus electric field, $E$, curves in HTS material [9] by extending those of percolation transition model (Yamafuji-Kiss model) [10], [11]. The expressions can quantitatively describe the $J-E$ curves in HTS conductor including not only their temperature dependence but also the magnetic anisotropy.

In this paper, we develop the optimal design code of the Bi-2223/Ag coil for the HTS-SMES by using the aforemen-

Manuscript received October 4, 2004. This work was supported by The Iwatani Naoji Foundations Research Grant, Mizuho Foundation for the Promotion of Sciences and the 21st century COE program (no. 14213201) in Japan.

K. Higashikawa, T. Nakamura, and T. Hoshino are with the Department of Electrical Engineering, Graduate School of Engineering, Kyoto University, Kyoto-Daigaku-Katsura, Nishikyo-Ku, Kyoto 615-8510, Japan (e-mail: kohei@asl.kuee.kyoto-u.ac.jp; tk_naka@kuee.kyoto-u.ac.jp; hoshino@asl.kuee.kyoto-u.ac.jp).

I. Muta is with the Saga University, 1 Honjo-cho, Saga 840-8502, Japan (e-mail: muta128@bb.excite.co.jp).

Digital Object Identifier 10.1109/TASC.2005.849326

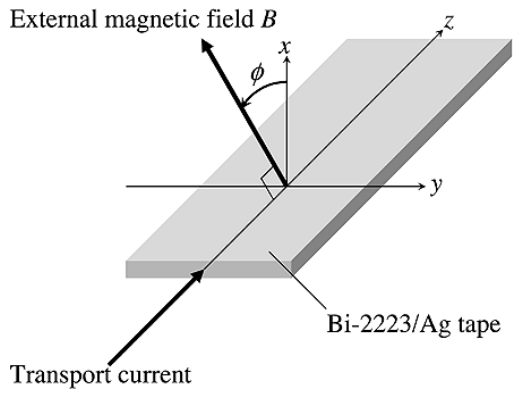

Fig. 1. Definition of the direction of the external magnetic field.

tioned expressions of current transport properties. The design is carried out by means of finite element method (FEM) and genetic algorithm (GA) [12], which is effective in case that the objective function has many local minimums. The winding geometry as well as the operating current of the coil is optimized for the minimization of its energy loss, on conditions of constant operating temperature, stored energy and total length of the conductor.

\section{Design Method}

\section{A. J-E Expressions}

The current density in HTS material, $J_{\mathrm{sc}}$, is calculated by the following expressions as functions of $E$, temperature, $T$, magnetic flux density, $B$, and its applied direction, $\phi$. The definition of the angle $\phi$ is illustrated in Fig. 1.

$$
\begin{aligned}
& J_{\mathrm{sc}}(E, T, B, \phi)= \\
& \left\{\begin{array}{lc}
J_{\mathrm{cm}}\left(T, B_{\text {caxis }}(B, \phi)\right) \cdot\left(\frac{m+1}{\rho_{\mathrm{FF}}} E J_{0}\left(T, B_{\text {caxis }}(B, \phi)\right)^{m}\right)^{\frac{1}{m+1}} \\
-\left|J_{\mathrm{cm}}\left(T, B_{\text {caxis }}(B, \phi)\right)\right| & \text { for } B_{\text {caxis }}(B, \phi) \leq B_{\mathrm{g}}(T, \phi) \\
\cdot\left(\frac{m+1}{\rho_{\mathrm{FF}}} E J_{0}\left(T, B_{\text {caxis }}(B, \phi)\right)^{m}+\left|J_{\mathrm{cm}}\left(T, B_{\text {caxis }}(B, \phi)\right)\right|^{m+1}\right)^{\frac{1}{m+1}} & \text { for } B_{\text {caxis }}(B, \phi)>B_{\mathrm{g}}(T, \phi)
\end{array}\right.
\end{aligned}
$$

where $B_{\mathrm{g}}$ is so-called glass-liquid transition magnetic flux density. A parameter $\rho_{\mathrm{FF}}$ is resistivity at uniform flux flow. $J_{\mathrm{cm}}$, $J_{0}$ and $m$ denote the minimum value, the half value of width and the parameter describing the shape of local critical current density distribution, respectively [9]-[11]. Furthermore, $B_{\text {caxis }}$ denotes equivalently perpendicular magnetic flux density, which is the effective c-axis component of the magnetic flux density, and expressed as follows [9]:

$$
B_{\text {caxis }}(B, \phi)=B\left(\cos ^{2} \phi+\frac{1}{\gamma^{2}} \sin ^{2} \phi\right)^{\frac{1}{2}}
$$


TABLE I

SPECIFICATIONS OF THE Bi-2223/Ag MULTIFILAMENTARY TAPE

\begin{tabular}{ll}
\hline \hline \multicolumn{1}{c}{ Items } & \multicolumn{1}{c}{ Values } \\
\hline Cross sectional width & $4.1 \mathrm{~mm}$ \\
Cross sectional thickness & $0.21 \mathrm{~mm}$ \\
Silver ratio & 2.8 \\
Critical temperature & $107 \mathrm{~K}$ \\
Critical current & $23.5 \mathrm{~A}$ (at $77.3 \mathrm{~K}$ and self field) \\
\hline \hline
\end{tabular}

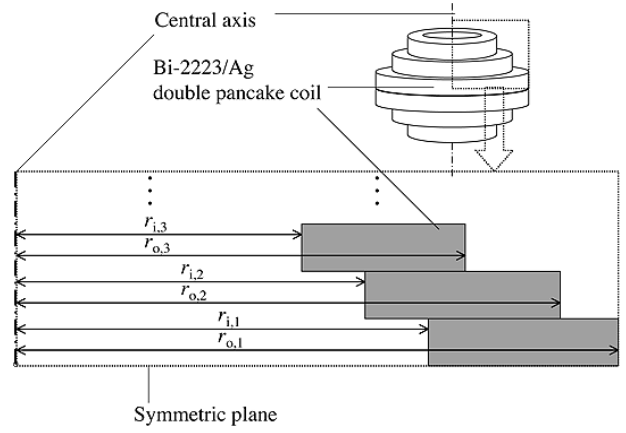

Fig. 2. Schematic diagram of the Bi-2223/Ag coil to be designed.

where $\gamma$ is the anisotropy ratio describing the misorientation of the grains in HTS material, and defined as follows [13]:

$$
\gamma=\frac{B_{\mathrm{g}}\left(T, 90^{\circ}\right)}{B_{\mathrm{g}}\left(T, 0^{\circ}\right)}
$$

As is already reported, expressions (1), (2) and (3) can quantitatively describe the current transport characteristics in Bi-2223/Ag multifilamentary tape [9].

The coil using the aforementioned $\mathrm{Bi}-2223 / \mathrm{Ag}$ tape is the subject for optimal design. Its specifications are listed in Table I. Silver ratio, $c$, is 2.8 , and then the overall $J$ in the tape can be expressed with consideration of the current sharing to the silver sheath in the following expression.

$$
J(E, T, B, \phi)=\frac{1}{1+c} J_{\mathrm{sc}}(E, T, B, \phi)+\frac{c}{1+c} J_{\mathrm{silver}}(E, T)
$$

where $J_{\text {silver }}$ is the current density in the sheath area, and can be expressed with the aid of Ohm's law as follows:

$$
J_{\text {silver }}(E, T)=\sigma_{\text {silver }}(T) E
$$

where $\sigma_{\text {silver }}$ is the temperature dependent electric conductivity of the silver sheath. Expression (4) is applied to FEM analysis.

\section{B. Model}

The diagram of the coil to be designed is shown in Fig. 2. That is, its schematic and the cross section of the tape's part are, respectively, illustrated in the upper and the lower parts of the figure. The coil is composed of multiple (the stack number, $\mathrm{N}$, is 6 in the figure) Bi-2223/Ag double pancake coils arranged coaxially, and the cross section of its upper half is modeled for FEM by using symmetric plane. Design variables $r_{\mathrm{i}, k}$ and $r_{\mathrm{o}, k}$ are correspondingly the inside and the outside radiuses of each double pancake coil, and $k$ denotes the ID number of the coil.

\section{Design Condition}

The design condition in this paper can be described as the following optimization problem.

minimize

$$
P\left(T, I, r_{\mathrm{i}, 1}, r_{\mathrm{o}, 1}, r_{\mathrm{i}, 2}, r_{\mathrm{o}, 2}, \ldots, r_{\mathrm{i}, \frac{N}{2}}, r_{\mathrm{o}, \frac{N}{2}}\right)
$$

subject to

$$
\begin{aligned}
& T=T_{\mathrm{op}}(=\text { const. }) \\
& W\left(I, r_{\mathrm{i}, 1}, r_{\mathrm{o}, 1}, r_{\mathrm{i}, 2}, r_{\mathrm{o}, 2}, \ldots, r_{\mathrm{i}, \frac{N}{2}}, r_{\mathrm{o}, \frac{N}{2}}\right)=W_{\mathrm{op}}(=\text { const. }) \\
& l\left(r_{\mathrm{i}, 1}, r_{\mathrm{o}, 1}, r_{\mathrm{i}, 2}, r_{\mathrm{o}, 2}, \ldots, r_{\mathrm{i}, \frac{N}{2}}, r_{\mathrm{o}, \frac{N}{2}}\right)=l_{\text {total }}(=\text { const. }) \\
& r_{\min } \leq r_{\mathrm{i}, k} \leq r_{\mathrm{o}, k} \leq r_{\max } \\
& r_{\mathrm{i}, k}, r_{\mathrm{o}, k}=r_{\min }+n \Delta r
\end{aligned}
$$

The following is the descriptions of the above expressions.

(6) $P$ is the loss of the coil, and can be expressed as functions of its operating temperature, $T$, operating current, $I$, and design variables $r_{\mathrm{i}, k}$ and $r_{\mathrm{o}, k}$ describing the winding geometry of the coil. The minimization of $P$ is the objective of the optimization.

(7) Constant $T_{\mathrm{op}}$ is the preset value of the operating temperature of the coil, and then $T$ remains to be constant under the optimization.

(8) The stored energy of the coil, $W$, is given as functions of $I, r_{\mathrm{i}, k}$ and $r_{\mathrm{o}, k}$, and they satisfy the condition that $W$ is equal to its preset value, $W_{\mathrm{op}}$. Design variables include $I$ along with $r_{\mathrm{i}, k}$ and $r_{\mathrm{o}, k}$ in order to satisfy such condition.

(9) The total length of the tape composing the coil, $l$, can be calculated with the use of $r_{\mathrm{i}, k}$ and $r_{\mathrm{o}, k}$, and they vary on condition that $l$ is equal to its preset value, $l_{\text {total }}$.

(10) Parameters $r_{\min }$ and $r_{\max }$ are, respectively, the lower and the higher limits of $r_{\mathrm{i}, k}$ and $r_{\mathrm{o}, k}$. The value of $r_{\min }$ is chosen to be large enough in order to avoid the influence of the mechanical strain on the $J-E$ curves in the tape. That is, the corresponding maximum strain factor is $0.210 \%$ with $r_{\min }=50.0 \mathrm{~mm}$. If $r_{\mathrm{i}, k}$ is equal to $r_{\mathrm{o}, k}$, the double pancake coil with ID number of $k$ does not exist.

(11) Design variables $r_{\mathrm{i}, k}$ and $r_{\mathrm{o}, k}$ vary at intervals, $\Delta r$, and $n$ is natural number. The value of $\Delta r$ is supposed to be the summation of the tape's thickness and the air gap length between adjacent turns. In this paper, however, such gap is not considered, and then $\Delta r$ is equal to the thickness of the tape.

\section{Calculation Procedure}

The optimal design of the coil is performed by means of GA and FEM as is already stated, and its calculation procedure is 


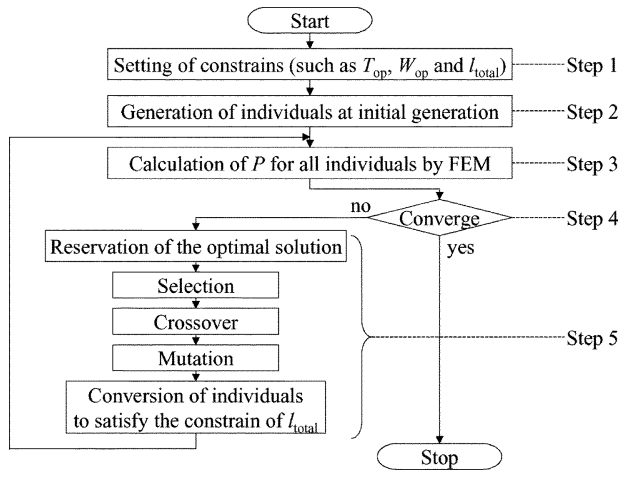

Fig. 3. Flowchart of the calculation procedure in the optimization.

illustrated in Fig. 3. The processes at each step in the figure are described as stated below.

Step 1: The values of $T_{\mathrm{op}}, W_{\mathrm{op}}$ and $l_{\text {total }}$ are determined

Step 2: The population with the number of individuals, $M$, at initial generation is generated. The individual means a set of the design variables such as $\left(I, r_{\mathrm{i}, 1}, r_{\mathrm{o}, 1}, r_{\mathrm{i}, 2}, r_{\mathrm{o}, 2}, \ldots, r_{\mathrm{i}, N / 2}, r_{\mathrm{o}, N / 2}\right)$.

Step 3: $\quad P$ is calculated for all individuals by FEM. The details at this step are described later (cf. steps 6-9).

Step 4: The convergence test is run. If the convergence condition is satisfied, the calculation is terminated, otherwise proceeds on to step 5 .

Step 5: The population at the next generation is generated. The method is as follows. Firstly, the optimal solution at the point is bequeathed to the next generation as it is. Secondly, the other $M-1$ individuals are generated according to the typical processes in GA such as selection, crossover and mutation. Thirdly, one of the parameters $r_{\mathrm{i}, k}$ and $r_{\mathrm{o}, k}$ chosen at random is converted to satisfy (9) with respect to such individuals. Lastly, the above optimal solution and $M-1$ individuals compose the population at the next generation.

The following is the detailed processes carried out for each individual at step 3 .

Step 6: The model for FEM analysis is arranged, that is, only the properties data are applied to the mesh created in advance. Therefore, it is not necessary to re-create the mesh during the calculation, and then the computation time is greatly reduced.

Step 7: $W_{\text {test }}$, which is the stored energy on condition that $I$ is equal to its test value, $I_{\text {test }}$, is computed by means of FEM.

Step 8: The operating current satisfying (8), $I_{\mathrm{op}}$, can be calculated by the following expression because $W$ is proportional to $I^{2}$.

$$
I_{\mathrm{op}}=I_{\text {test }}\left(\frac{W_{\mathrm{op}}}{W_{\text {test }}}\right)^{\frac{1}{2}} .
$$

Step 9: $\quad P$ with $I=I_{\mathrm{op}}$ is computed by means of FEM. $P$ is defined as the spatial integration of the inner product of electric field and current density.
TABLE II

The OptIMAL SOLUTION ON CONDITION A

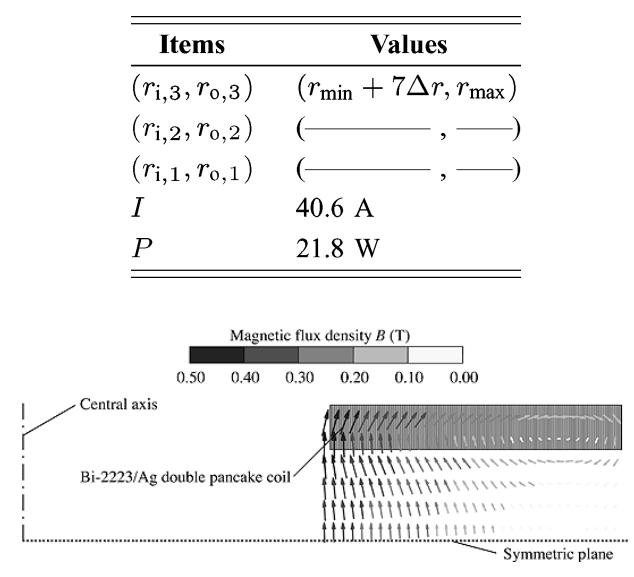

Fig. 4. The optimal shape and magnetic field distribution on condition A.

TABLE III

AN EXAMPLE SOLUTION ON CONDITION A

\begin{tabular}{ll}
\hline \hline \multicolumn{1}{c}{ Items } & \multicolumn{1}{c}{ Values } \\
\hline$\left(r_{\mathrm{i}, 3}, r_{\mathrm{o}, 3}\right)$ & $(-,-)$ \\
$\left(r_{\mathrm{i}, 2}, r_{\mathrm{o}, 2}\right)$ & $(-,-)$ \\
$\left(r_{\mathrm{i}, 1}, r_{\mathrm{o}, 1}\right)$ & $\left(r_{\min }+7 \Delta r, r_{\max }\right)$ \\
$I$ & $35.1 \mathrm{~A}$ \\
$P$ & $29.3 \mathrm{~W}$ \\
\hline \hline
\end{tabular}

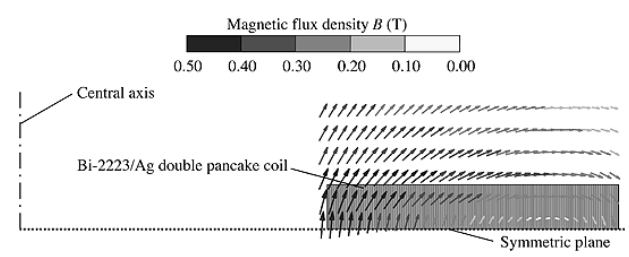

Fig. 5. An example shape and magnetic field distribution on condition A.

\section{RESULTS AND DISCUSSION}

\section{A. Condition A}

Table II and Fig. 4 respectively show the optimal solution and the optimal shape with the magnetic flux density distribution at $T_{\text {op }}=65.0 \mathrm{~K}, W_{\text {op }}=100 \mathrm{~J}, l_{\text {total }}=500 \mathrm{~m}$ and $N=6$ (defined as Condition A). As shown in the figure, the optimal shape is the configuration of only one double pancake coil at $k=3$. That is, the coil is located farthest away from the symmetry plane. On the other hand, the configuration of one double pancake coil at $k=1$, i.e., placed closest to the symmetry plane, is also considered for example, and the corresponding variables and the shape with the magnetic flux density distribution are shown in Table III and Fig. 5, respectively. As can be seen by comparison, the intensity of magnetic flux penetrating into the coil in Fig. 5 is larger than that in Fig. 4. Furthermore, the normal component of magnetic flux density with respect to the tape's broad surface in Fig. 5 is also larger than that in Fig. 4. The reason is that the performance of the coil in Fig. 5 is more largely influenced by the coil located below the symmetry plane compared to that in Fig. 4. Therefore, the result of the optimal design at Condition $\mathrm{A}$ is the configuration of 2 double pancake coils 
TABLE IV

THE Optimal SOLUTION ON CONDITION B

\begin{tabular}{ll}
\hline \hline \multicolumn{1}{c}{ Items } & \multicolumn{1}{c}{ Values } \\
\hline$\left(r_{\mathrm{i}, 3}, r_{\mathrm{o}, 3}\right)$ & $\left(r_{\min }+2 \Delta r, r_{\max }\right)$ \\
$\left(r_{\mathrm{i}, 2}, r_{\mathrm{o}, 2}\right)$ & $\left(r_{\min }+220 \Delta r, r_{\max }\right)$ \\
$\left(r_{\mathrm{i}, 1}, r_{\mathrm{o}, 1}\right)$ & $\left(r_{\min }+45 \Delta r, r_{\max }\right)$ \\
$I$ & $19.1 \mathrm{~A}$ \\
$P$ & $309 \mathrm{~mW}$ \\
\hline \hline
\end{tabular}

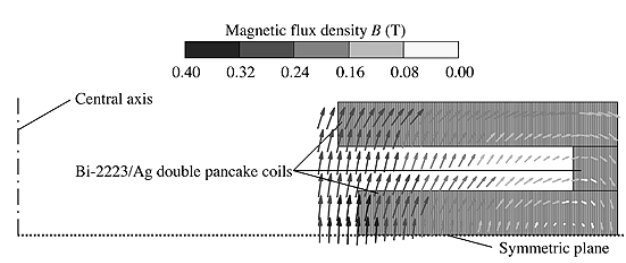

Fig. 6. The optimal shape and magnetic field distribution on condition B.

TABLE V

THE OPTIMAL SOLUTION ON CONDITION C

\begin{tabular}{ll}
\hline \hline \multicolumn{1}{c}{ Items } & \multicolumn{1}{c}{ Values } \\
\hline$\left(r_{\mathrm{i}, 4}, r_{\mathrm{o}, 4}\right)$ & $\left(r_{\min }+12 \Delta r, r_{\max }\right)$ \\
$\left(r_{\mathrm{i}, 3}, r_{\mathrm{o}, 3}\right)$ & $\left(r_{\min }+223 \Delta r, r_{\max }\right)$ \\
$\left(r_{\mathrm{i}, 2}, r_{\mathrm{o}, 2}\right)$ & $\left(r_{\min }+168 \Delta r, r_{\max }\right)$ \\
$\left(r_{\mathrm{i}, 1}, r_{\mathrm{o}, 1}\right)$ & $\left(r_{\min }+35 \Delta r, r_{\max }\right)$ \\
$I$ & $16.6 \mathrm{~A}$ \\
$P$ & $83.8 \mathrm{~mW}$ \\
\hline
\end{tabular}

arranged away from each other (because the symmetry plane is considered), even if comparatively large operating current is required, i.e., $I=40.6$ A for Fig. 4 and 35.1 A for Fig. 5.

\section{B. Condition B}

It is not always possible to apply entire length of the conductor only to one double pancake coil as shown in Fig. 4. As one of such case study, the optimal design is performed at $T_{\text {op }}=65.0 \mathrm{~K}, W_{\text {op }}=100 \mathrm{~J}, l_{\text {total }}=1000 \mathrm{~m}$ and $N=6$ (defined as Condition B). The results of the variables and the shape with the magnetic flux density distribution are shown in Table IV and Fig. 6, respectively. As shown in the figure, the double pancake coil at $k=3$ is the largest. That can be illustrated with the above discussion in Condition A. The second largest is the coil at $k=1$. The reason is considered to be the following two things. One is that coils should be arranged away from each other as stated in Condition A. Therefore, the coil at $k=1$ is larger than that at $k=2$ because the former is located farther from the largest coil at $k=3$ than the latter. The other is that magnetic flux is applied almost parallel to the broad surface of the tape near the symmetric plane, and then corresponding energy loss is reduced due to less effect of the magnetic anisotropy of the tape. According to such reasons, the optimal shape at Condition B is as shown in Fig. 6.

\section{Condition C}

The optimal design is also carried out at $T_{\mathrm{op}}=65.0 \mathrm{~K}$, $W_{\text {op }}=100 \mathrm{~J}, l_{\text {total }}=1200 \mathrm{~m}$ and $N=8$, and the results

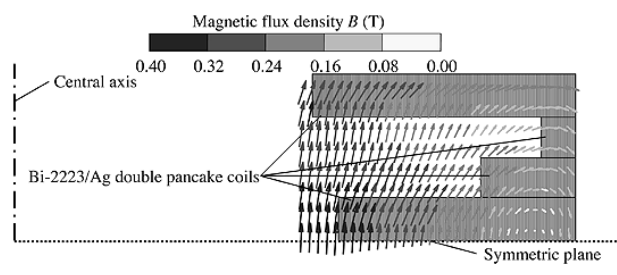

Fig. 7. The optimal shape and magnetic field distribution on condition C.

of the variables and the shape with the magnetic flux density distribution are shown in Table V and Fig. 7, respectively. The discussion is same as stated in Condition B.

\section{CONCLUSION}

We designed Bi-2223/Ag coils for SMES based on GA and FEM with consideration of the quantitative $J-E$ expressions in the tape. As a result, we found that the double pancake coils should be arranged away from each other. The attractiveness of coil separation in the solenoidal array suggests that separations found in a toroidal array may be attractive as well. Evaluations of toroidal configurations are planned as future investigations.

\section{REFERENCES}

[1] K. Higashikawa, T. Nakamura, I. Muta, and T. Hoshino, "Investigation of dissipative behavior in $\mathrm{Bi}-2223 / \mathrm{Ag}$ coil with consideration of multifilamentary structure," in Proc. EUCAS'03 (in press), Sorrento Napoli, Italy, 2003.

[2] - "Anisotropic distributions of current density and electric field in Bi-2223/Ag coil with consideration of multifilamentary structure" (in accepted), Physica $C$.

[3] A. Korpela, J. Lehtonen, and R. Mikkonen, "Optimization of HTS superconducting magnetic energy storage magnet volume," Supercond. Sci. Technol., vol. 16, pp. 833-837, 2003.

[4] M. Yamaguchi, A. Honma, S. Ishiguri, S. Fukui, I. Muta, and T. Nakamura, "A study on performance improvements of HTS coil," IEEE Trans. Appl. Supercond., vol. 13, no. 2, pp. 1848-1851, 2003.

[5] S. Noguchi, H. Yamashita, and A. Ishiyama, "An optimization method for design of SMES coils using YBCO tape," IEEE Trans. Appl. Supercond., vol. 13, no. 2, pp. 1856-1859, 2003.

[6] - "An optimal design method for SMES coils using HTS tapes," IEEE Trans. Appl. Supercond., vol. 12, no. 1, pp. 1459-1462, 2002.

[7] J. H. Kim, S. Hahn, C. H. Im, J. K. Kim, H. K. Jung, and S. Hahn, "Design of a 200-kJ HTS SMES system," IEEE Trans. Appl. Supercond., vol. 12, no. 1, pp. 774-777, 2002.

[8] J. Pitel, P. Kovac, A. Kasztler, and H. Kirchmayr, "Optimization of winding geometry of $\mathrm{Bi}(2223) \mathrm{Ag}$ coils with respect to external magnetic field," IEEE Trans. Appl. Supercond., vol. 11, no. 1, pp. 2324-2327, 2001.

[9] T. Nakamura, S. Tsuchiya, A. Fujio, T. Hoshino, and I. Muta, "Angular dependence of E-J characteristics and dissipative properties in Bi-2223/Ag tape," Supercond. Sci. Technol., vol. 15, pp. 230-235, 2002.

[10] T. Kiss, T. Nakamura, N. Mishiro, K. Hasegawa, M. Inoue, M. Takeo, F. Irie, and K. Yamafuji, "Transport characteristics in high Tc superconductors," in Proc. MT15 (no. 2), 1998, pp. 1052-1055.

[11] T. Kiss, K. Hasegawa, M. Inoue, M. Takeo, H. Okamoto, and F. Irie, "Critical current properties in high Tc superconductors," Cryogenic Engineering (Teion-Kōgaku) (in Japanese), vol. 34, no. 7, pp. 322-331, 1999.

[12] D. E. Goldberg, Genetic Algorithm in Search, Optimization and Machine Learning. Reading, MA: Addison-Wesley, 1989.

[13] T. Nakamura, T. Yamamoto, S. Tsuchiya, A. Fujio, T. Hoshino, I. Muta, S. S. Oh, and H. S. Ha, "Anisotropy of critical current and glass-liquid transition flux density in Bi-2223/Ag tape," Supercond. Sci. Technol., vol. 13 , pp. 1521-1525, 2000. 